\title{
Adaptive Wave Models for Sophisticated Option Pricing
}

\author{
Vladimir G. Ivancevic \\ Defence Science \& Technology Organisation, Canberra, Australia \\ E-mail: Vladimir.Ivancevic@dsto.defence.gov.au \\ Received August 19, 2011; revised October 13, 2011; accepted October 22, 2011
}

\begin{abstract}
Adaptive wave model for financial option pricing is proposed, as a high-complexity alternative to the standard Black-Scholes model. The new option-pricing model, representing a controlled Brownian motion, includes two wave-type approaches: nonlinear and quantum, both based on (adaptive form of) the Schrödinger equation. The nonlinear approach comes in two flavors: for the case of constant volatility, it is defined by a single adaptive nonlinear Schrödinger (NLS) equation, while for the case of stochastic volatility, it is defined by an adaptive Manakov system of two coupled NLS equations. The linear quantum approach is defined in terms of de Broglie's plane waves and free-particle Schrödinger equation. In this approach, financial variables have quantum-mechanical interpretation and satisfy the Heisenberg-type uncertainty relations. Both models are capable of successful fitting of the Black-Scholes data, as well as defining Greeks.
\end{abstract}

Keywords: Black-Scholes Option Pricing, Adaptive Nonlinear Schrödinger Equation, Adaptive Manakov System, Quantum-Mechanical Option Pricing, Market-Heat Potential

\section{Introduction}

Recall that the celebrated Black-Scholes partial differential equation (PDE) describes the time-evolution of the market value of a stock option [1,2]. Formally, for a function $u=u(t, s)$ defined on the domain

$0 \leq s<\infty, 0 \leq t \leq T$ and describing the market value of a stock option with the stock (asset) price $s$, the BlackScholes PDE can be written (using the physicist notation: $\left.\partial_{z} u=\partial u / \partial z\right)$ as a diffusion-type equation:

$$
\partial_{t} u=-\frac{1}{2}(\sigma s)^{2} \partial_{s s} u-r s \partial_{s} u+r u,
$$

where $\sigma>0$ is the standard deviation, or volatility of $s$, $r$ is the short-term prevailing continuously-compounded risk - free interest rate, and $T>0$ is the time to maturity of the stock option. In this formulation it is assumed that the underlying (typically the stock) follows a geometric Brownian motion with "drift" $\mu$ and volatility $\sigma$, given by the stochastic differential equation (SDE) [3]

$$
\mathrm{d} s(t)=\mu s(t) \mathrm{d} t+\sigma s(t) \mathrm{d} W(t),
$$

where $W$ is the standard Wiener process. The Black-Scholes PDE (1) is usually derived from SDEs describing the geometric Brownian motion (2), with the stock-price solution given by:

$$
s(t)=s(0) \mathrm{e}^{\left(\mu-\frac{1}{2} \sigma^{2}\right) t+\sigma W(t)} .
$$

In mathematical finance, derivation is usually performed using Itô lemma [4] (assuming that the underlying asset obeys the Itô SDE), while in physics it is performed using Stratonovich interpretation $[5,6]$ (assuming that the underlying asset obeys the Stratonovich SDE [8]).

The Black-Sholes PDE (1) can be applied to a number of one-dimensional models of interpretations of prices given to $u$, e.g., puts or calls, and to $s$, e.g., stocks or futures, dividends, etc. The most important examples are European call and put options, defined by:

$$
\begin{gathered}
u_{\text {Call }}(s, t)=s \mathcal{N}\left(\mathrm{d}_{1}\right) \mathrm{e}^{-T \delta}-k \mathcal{N}\left(\mathrm{d}_{2}\right) \mathrm{e}^{-r T}, \\
u_{\text {Put }}(s, t)=k \mathcal{N}\left(-\mathrm{d}_{2}\right) \mathrm{e}^{-r T}-s \mathcal{N}\left(-\mathrm{d}_{1}\right) \mathrm{e}^{-T \delta}, \\
\mathcal{N}(\lambda)=\frac{1}{2}\left(1+\operatorname{erf}\left(\frac{\lambda}{\sqrt{2}}\right)\right), \\
\mathrm{d}_{1}=\frac{\ln \left(\frac{s}{k}\right)+T\left(r-\delta+\frac{\sigma^{2}}{2}\right)}{\sigma \sqrt{T}}, \\
\mathrm{~d}_{2}=\frac{\ln \left(\frac{s}{k}\right)+T\left(r-\delta-\frac{\sigma^{2}}{2}\right)}{\sigma \sqrt{T}},
\end{gathered}
$$


where $\operatorname{erf}(\lambda)$ is the (real-valued) error function, $k$ denotes the strike price and $\delta$ represents the dividend yield. In addition, for each of the call and put options, there are five Greeks (see, e.g. [9,10]), or sensitivities, which are partial derivatives of the option-price with respect to stock price (Delta), interest rate (Rho), volatility (Vega), elapsed time since entering into the option (Theta), and the second partial derivative of the option-price with respect to the stock price (Gamma).

Using the standard Kolmogorov probability approach, instead of the market value of an option given by the Black-Scholes Equation (1), we could consider the corresponding probability density function (PDF) given by the backward Fokker-Planck equation (see [6,7]). Alternatively, we can obtain the same PDF (for the market value of a stock option), using the quantum-probability formalism [11,12], as a solution to a time-dependent linear or nonlinear Schrödinger equation for the evolution of the complex-valued wave $\psi$-function for which the absolute square, $|\psi|^{2}$, is the PDF. The adaptive nonlinear Schrödinger (NLS) equation was recently used in [10] as an approach to option price modelling, as briefly reviewed in this section. The new model, philosophically founded on adaptive markets hypothesis $[13,14]$ and Elliott wave market theory $[15,16]$, as well as my own recent work on quantum congition $[17,18]$, describes adaptively controlled Brownian market behavior. This nonlinear approach to option price modelling is reviewed in the next section. Its important limiting case with low interest-rate reduces to the linear Schrödinger equation. This linear approach to option price modelling is elaborated in the subsequent section.

\section{Nonlinear Adaptive Wave Model for General Option Pricing}

\subsection{Adaptive NLS Model}

The adaptive, wave-form, nonlinear and stochastic optionpricing model with stock price $s$, volatility $\sigma$ and interest rate $r$ is formally defined as a complex-valued, focusing $(1+1)-N L S$ equation, defining the time-dependent option-price wave function $\psi=\psi(s, t)$, whose absolute square $|\psi(s, t)|^{2}$ represents the probability density function (PDF) for the option price in terms of the stock price and time. In natural quantum units, this NLS equation reads:

$$
i \partial_{t} \psi=-\frac{1}{2} \sigma \partial_{s s} \psi-\beta|\psi|^{2} \psi, \quad(i=\sqrt{-1}),
$$

where $\beta=\beta(r, w)$ denotes the adaptive market-heat potential (see [19]), so the term $V(\psi)=-\beta|\psi|^{2}$ represents the $\psi$-dependent potential field. In the simplest nonadaptive scenario $\beta$ is equal to the interest rate $r$, while in the adaptive case it depends on the set of adjustable synaptic weights $\left\{w_{j}^{i}\right\}$ as:

$$
\beta(r, w)=r \sum_{i=1}^{n} w_{1}^{i} \operatorname{erf}\left(\frac{w_{2}^{i} s}{w_{3}^{i}}\right) .
$$

Physically, the NLS Equation (5) describes a nonlinear wave (e.g. in Bose-Einstein condensates) defined by the complex-valued wave function $\psi(s, t)$ of real space and time parameters. In the present context, the space-like variable $s$ denotes the stock (asset) price.

The NLS Equation (5) has been exactly solved using the power series expansion method [20,21] of Jacobi elliptic functions [22]. Consider the $\psi$-function describing a single plane wave, with the wave number $k$ and circular frequency $\omega$ :

$$
\psi(s, t)=\varphi(\xi) \mathrm{e}^{\mathrm{i}(k s-\omega t)},
$$

with

$$
\xi=s-\sigma k t \text { and } \varphi(\xi) \in \mathbb{R} .
$$

Its substitution into the NLS Equation (5) gives the nonlinear oscillator ODE:

$$
\varphi^{\prime \prime}(\xi)+\left[\omega-\frac{1}{2} \sigma k^{2}\right] \varphi(\xi)+\beta \varphi^{3}(\xi)=0 .
$$

We can seek a solution $\varphi(\xi)$ for (8) as a linear function [21]

$$
\varphi(\xi)=a_{0}+a_{1} \operatorname{sn}(\xi)
$$

where $\operatorname{sn}(s)=\operatorname{sn}(s, m)$ are Jacobi elliptic sine functions with elliptic modulus $m \in[0,1]$, such that $\operatorname{sn}(s, 0)=\sin (s)$ and $\operatorname{sn}(s, 1)=\tanh (s)$. The solution of (8) was calculated in [10] to be

$$
\begin{gathered}
\varphi(\xi)= \pm m \sqrt{\frac{-\sigma}{\beta}} \operatorname{sn}(\xi), \text { for } m \in[0,1], \\
\varphi(\xi)= \pm \sqrt{\frac{-\sigma}{\beta}} \tanh (\xi), \text { for } m=1
\end{gathered}
$$

This gives the exact periodic solution of (5) as [10]

$$
\psi_{1}(s, t)= \pm m \sqrt{\frac{-\sigma}{\beta(w)}} \operatorname{sn}(s-\sigma k t) \mathrm{e}^{\mathrm{i}\left[k s-\frac{1}{2} \sigma t\left(1+m^{2}+k^{2}\right)\right]}
$$

for $m \in[0,1)$;

$$
\psi_{2}(s, t)= \pm \sqrt{\frac{-\sigma}{\beta(w)}} \tanh (s-\sigma k t) \mathrm{e}^{\mathrm{i}\left[k s-\frac{1}{2} \sigma t\left(2+k^{2}\right)\right]},
$$

for $m=1$,

where (9) defines the general solution, while (10) defines 
the envelope shock-wave (or, "dark soliton") solution of the NLS Equation (5).

Alternatively, if we seek a solution $\varphi(\xi)$ as a linear function of Jacobi elliptic cosine functions, such that $\operatorname{cn}(s, 0)=\cos (s)$ and $\operatorname{cn}(s, 1)=\operatorname{sech}(s)^{2}$,

$$
\varphi(\xi)=a_{0}+a_{1} \operatorname{cn}(\xi)
$$

then we get [10]

$$
\psi_{3}(s, t)= \pm m \sqrt{\frac{\sigma}{\beta(w)}} \operatorname{cn}(s-\sigma k t) \mathrm{e}^{\mathrm{i}\left[k s-\frac{1}{2} \sigma t\left(1-2 m^{2}+k^{2}\right)\right]},
$$

for $m \in[0,1)$;

$$
\begin{aligned}
& \psi_{4}(s, t)= \pm \sqrt{\frac{\sigma}{\beta(w)}} \operatorname{sech}(s-\sigma k t) \mathrm{e}^{i\left[k s-\frac{1}{2} \sigma t\left(k^{2}-1\right)\right]}, \\
& \text { for } m=1
\end{aligned}
$$

where (11) defines the general solution, while (12) defines the envelope solitary-wave (or, "bright soliton") solution of the NLS Equation (5).

In all four solution Expressions (9), (10), (11) and (12), the adaptive potential $\beta(w)$ is yet to be calculated using either unsupervised Hebbian learning, or supervised Levenberg-Marquardt algorithm (see, e.g. [23,24]). In this way, the NLS Equation (5) becomes the quantum neural network (see [18]). Any kind of numerical analysis can be easily performed using above closed-form solutions $\psi_{i}(s, t)(i=1, \cdots, 4)$ as initial conditions.

The adaptive NLS-PDFs of the shock-wave type (10) has been used in [10] to fit the Black-Scholes call and put options (see Figures 1 and 2). Specifically, the adaptive heat potential (6) was combined with the spatial part of $(10)$

$$
\varphi(s)=\left|\sqrt{\frac{\sigma}{\beta}} \tanh (s-k t \sigma)\right|^{2},
$$

while parameter estimates where obtained using 100 iterations of the Levenberg-Marquardt algorithm.

\footnotetext{
${ }^{1}$ A shock wave is a type of fast-propagating nonlinear disturbance that carries energy and can propagate through a medium (or, field). It is characterized by an abrupt, nearly discontinuous change in the characteristics of the medium. The energy of a shock wave dissipates relatively quickly with distance and its entropy increases. On the other hand, a soliton is a self-reinforcing nonlinear solitary wave packet that maintains its shape while it travels at constant speed. It is caused by a cancelation of nonlinear and dispersive effects in the medium (or, field).

${ }^{2}$ A closely related solution of an anharmonic oscillator ODE:

$$
\varphi^{\prime \prime}(s)+\varphi(s)+\varphi^{3}(s)=0
$$

is given by

$$
\varphi(s)=\sqrt{\frac{2 m}{1-2 m}} \mathrm{cn}\left(\sqrt{1+\frac{2 m}{1-2 m}} \mathrm{~s}, m\right) .
$$

${ }^{3}$ Controlled stochastic volatility here represents volatility evolving in a stochastic manner but within the controlled boundaries.
}

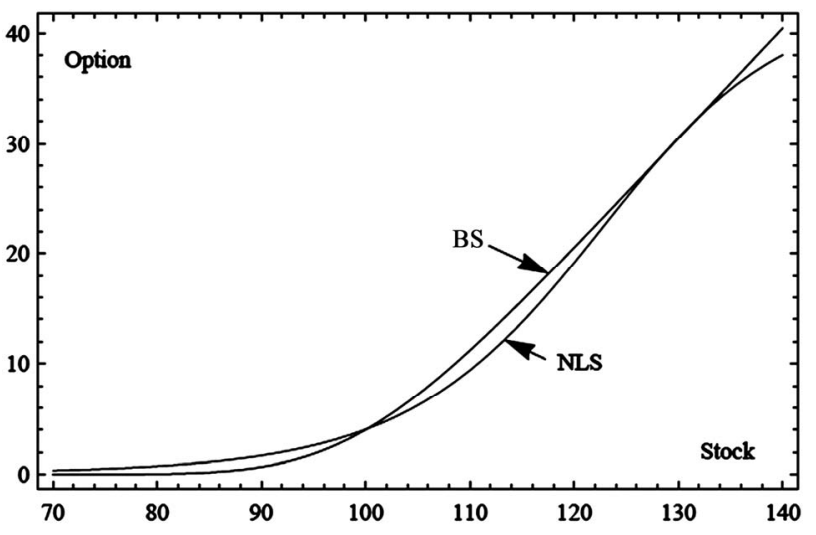

Figure 1. Fitting the Black-Scholes call option with (w)-adaptive PDF of the shock-wave NLS-solution (10).

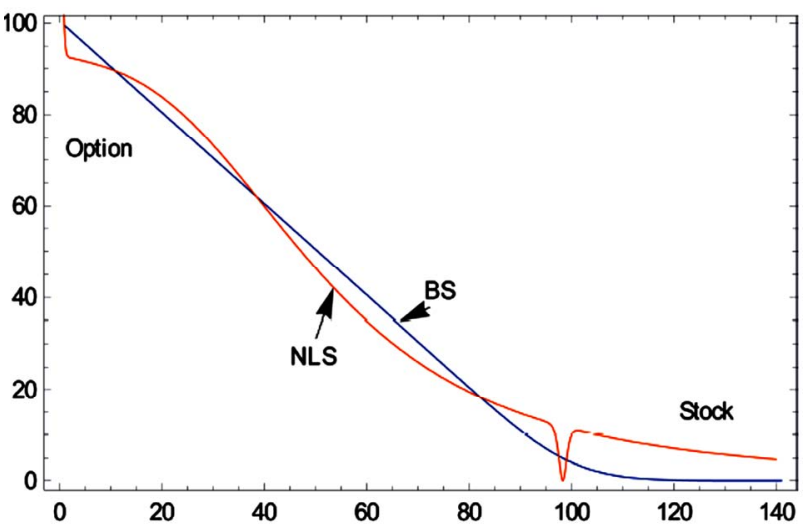

Figure 2. Fitting the Black-Scholes put option with (w)-adaptive PDF of the shock-wave NLS 2(s, $t$ ) solution (10). Notice the kink near $s=100$.

As can be seen from Figure 2 there is a kink near $s=100$. This kink, which is a natural characteristic of the spatial shock-wave (13), can be smoothed out (Figure 3) by taking the sum of the spatial parts of the shock-wave solution (10) and the soliton solution (12) as:

$$
\begin{aligned}
& \varphi(s) \\
= & \left|\sqrt{\frac{\sigma}{\beta}}\left[\mathrm{d}_{1} \tanh (s-k t \sigma)+\mathrm{d}_{2} \operatorname{sech}(s-k t \sigma)\right]\right|^{2} .
\end{aligned}
$$

The adaptive NLS-based Greeks (Delta, Rho, Vega, Theta and Gamma) have been defined in [10], as partial derivatives of the shock-wave solution (10).

\subsection{Adaptive Manakov System}

Next, for the purpose of including a controlled stochastic volatility $^{3}$ into the adaptive-NLS Model (5), the full bidirectional quantum neural computation model [18] for option-price forecasting has been formulated in [10] as a self-organized system of two coupled self-focusing NLS 


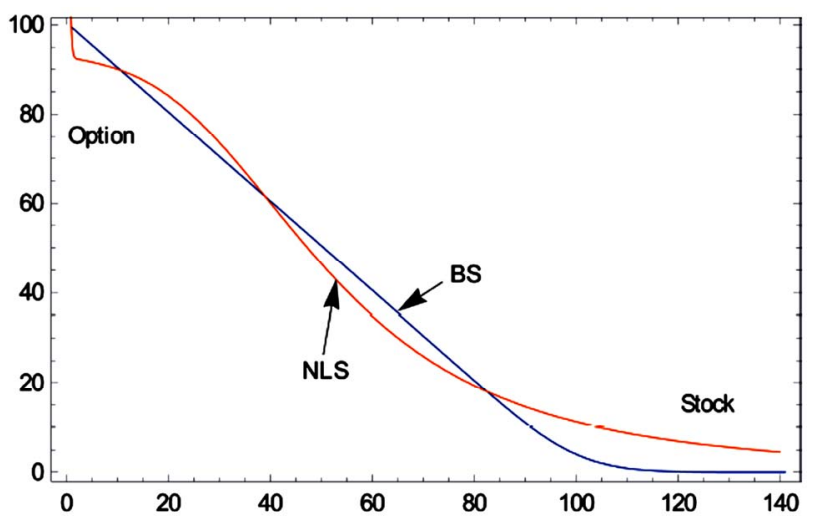

Figure 3. Smoothing out the kink in the put option fit, by combining the shock-wave solution with the soliton solution, as defined by (14).

equations: one defining the option-price wave function $\psi=\psi(s, t)$ and the other defining the volatility wave function $\sigma=\sigma(s, t)$ :

Volatility NLS:

$\mathrm{i} \partial_{t} \sigma=-\frac{1}{2} \partial_{s s} \sigma-\beta(r, w)\left(|\sigma|^{2}+|\psi|^{2}\right) \sigma$

Option price NLS:

$$
\mathrm{i} \partial_{t} \psi=-\frac{1}{2} \partial_{s s} \psi-\beta(r, w)\left(|\sigma|^{2}+|\psi|^{2}\right) \psi .
$$

In this coupled model, the $\sigma$-NLS (15) governs the $(s, t)$-evolution of stochastic volatility, which plays the role of a nonlinear coefficient in (16); the $\psi$-NLS (16) defines the $(s, t)$-evolution of option price, which plays the role of a nonlinear coefficient in (15). The purpose of this coupling is to generate a leverage effect, i.e. stock volatility is (negatively) correlated to stock returns ${ }^{4}$ (see, e.g. [27]). This bidirectional associative memory effectively performs quantum neural computation [18], by giving a spatio-temporal and quantum generalization of Kosko's BAM family of neural networks [28,29]. In addition, the shock-wave and solitary-wave nature of the coupled NLS equations may describe brain-like effects frequently occurring in financial markets: volatility/price propagation, reflection and collision of shock and solitary waves (see [30]).

The coupled NLS-system (15)-(16), without an embedded $w$-learning (i.e., for constant $\beta=r$-the interest rate), actually defines the well-known Manakov system, ${ }^{5}$ proven by S. Manakov in 1973 [31] to be completely integrable, by the existence of infinite number of

${ }^{4}$ The hypothesis that financial leverage can explain the leverage effect was first discussed by F. Black [26].

${ }^{5}$ Manakov system has been used to describe the interaction between wave packets in dispersive conservative media, and also the interaction between orthogonally polarized components in nonlinear optical fibres (see, e.g. [32, 33] and references therein). involutive integrals of motion. It admits "bright" and "dark" soliton solutions. The simplest solution of (15)-(16), the so-called Manakov bright 2-soliton, has the form resembling that of the sech-solution (12) (see [34-40]), and is formally defined by:

$$
\psi_{\text {sol }}(s, t)=2 b \operatorname{csech}(2 b(s+4 a t)) \mathrm{e}^{-2 \mathrm{i}\left(2 a^{2} t+a s-2 b^{2} t\right)},
$$

where $\psi_{\text {sol }}(s, t)=\left(\begin{array}{l}\sigma(s, t) \\ \psi(s, t)\end{array}\right), \mathbf{c}=\left(c_{1}, c_{2}\right)^{T}$ is a unit vector such that $\left|c_{1}\right|^{2}+\left|c_{2}\right|^{2}=1$.

Real-valued parameters $a$ and $b$ are some simple functions of $(\sigma, \beta, k)$, which can be determined by the Levenberg-Marquardt algorithm. I have argued in [10] that in some short-time financial situations, the adaptation effect on $\beta$ can be neglected, so our option-pricing Model (15)-(16) can be reduced to the Manakov 2-soliton Model (17), as depicted and explained in Figure 4.

\section{Quantum Wave Model for Low Interest-Rate Option Pricing}

In the case of a low interest-rate $r \ll 1$, we have $\beta(r) \ll 1$, so $V(\psi) \rightarrow 0$, and therefore Equation (5) can be approximated by a quantum-like option wave packet. It is defined by a continuous superposition of de Broglie's plane waves, 'physically' associated with a free quantum particle of unit mass. This linear wave packet, given by the time-dependent complex-valued wave function $\psi=\psi(s, t)$, is a solution of the linear Schrödinger equation with zero potential energy, Hamiltonian operator $\hat{H}$ and volatility $\sigma$ playing the role similar to the Planck constant. This equation can be written as:

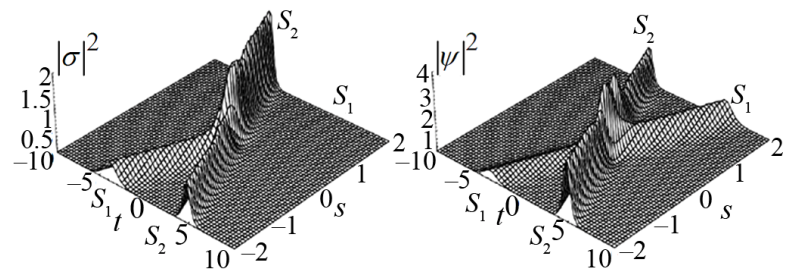

Figure 4. Hypothetical market scenario including sample PDFs for volatility $|\sigma|^{2}$ and $|\psi|^{2}$ of the Manakov 2-soliton (17). On the left, we observe the $(s, t)$ - evolution of stochastic volatility: we have a collision of two volatility component-solitons, $S_{1}(s, t)$ and $S_{2}(s, t)$, which join together into the resulting soliton $S_{2}(s, t)$, annihilating the $S_{1}(s, t)$ component in the process. On the right, we observe the $(s, t)$-evolution of option price: we have a collision of two option component-solitons, $S_{1}(s, t)$ and $S_{2}(s, t)$, which pass through each other without much change, except at the collision point. Due to symmetry of the Manakov system, volatility and option price can exchange their roles. 


$$
\mathrm{i} \sigma \partial_{t} \psi=\hat{H} \psi, \text { where } \hat{H}=-\frac{\sigma^{2}}{2} \partial_{s s} .
$$

Thus, we consider the $\psi$-function describing a single de Broglie's plane wave, with the wave number $k$, linear momentum $p=\sigma k$, wavelength $\lambda_{k}=2 \pi / k$, angular frequency $\omega_{k}=\sigma k^{2} / 2$, and oscillation period $T_{k}=2 \pi / \omega_{k}=4 \pi / \sigma k^{2}$. It is defined by (compare with $[41,42,12])$

$$
\begin{aligned}
\psi_{k}(s, t) & =A \mathrm{e}^{\mathrm{i}\left(k s-\omega_{k} t\right)}=A \mathrm{e}^{\mathrm{i}\left(k s-\frac{\sigma k^{2}}{2} t\right)} \\
& =A \cos \left(k s-\frac{\sigma k^{2}}{2} t\right)+A i \sin \left(k s-\frac{\sigma k^{2}}{2} t\right)
\end{aligned}
$$

where $A$ is the amplitude of the wave, the angle $\left(k s-\omega_{k} t\right)=\left(k s-\frac{\sigma k^{2}}{2} t\right)$ represents the phase of the wave $\psi_{k}$ with the phase velocity: $v_{k}=\omega_{k} / k=\sigma k / 2$. The space-time wave function $\psi(s, t)$ that satisfies the linear Schrödinger Equation (18) can be decomposed (using Fourier's separation of variables) into the spatial part $\varphi(s)$ and the temporal part $\mathrm{e}^{-\mathrm{i} \omega t}$ as:

$$
\psi(s, t)=\varphi(s) \mathrm{e}^{-\mathrm{i} \omega t}=\varphi(s) \mathrm{e}^{-\frac{\mathrm{i}}{\sigma} E t} .
$$

The spatial part, representing stationary (or, amplitude) wave function, $\varphi(s)=A \mathrm{e}^{\mathrm{i} k s}$, satisfies the linear harmonic oscillator, which can be formulated in several equivalent forms:

$$
\begin{aligned}
& \varphi^{\prime \prime}+k^{2} \varphi=0, \quad \varphi^{\prime \prime}+\left(\frac{p}{\sigma}\right)^{2} \varphi=0, \\
& \varphi^{\prime \prime}+\left(\frac{\omega_{k}}{v_{k}}\right)^{2} \varphi=0, \quad \varphi^{\prime \prime}+\frac{2 E_{k}}{\sigma^{2}} \varphi=0 .
\end{aligned}
$$

Planck's energy quantum of the option wave $\psi_{k}$ is given by: $E_{k}=\sigma \omega_{k}=\frac{1}{2}(\sigma k)^{2}$.

From the plane-wave expressions (19) we have:

$$
\psi_{k}(s, t)=A \mathrm{e}^{\frac{\mathrm{i}}{\sigma}\left(p s-E_{k} t\right)}
$$

-for the wave going to the "right" and

$$
\psi_{k}(s, t)=A \mathrm{e}^{-\frac{\mathrm{i}}{\sigma}\left(p s+E_{k} t\right)}
$$

-for the wave going to the "left".

The general solution to (18) is formulated as a linear combination of de Broglie's option waves (19), comprising the option wave-packet:

$$
\psi(s, t)=\sum_{i=0}^{n} c_{i} \psi_{k_{i}}(s, t), \quad(\text { with } n \in \mathbb{N}) .
$$

Its absolute square, $|\psi(s, t)|^{2}$, represents the probability density function at a time $t$.

The group velocity of an option wave-packet is given by: $v_{g}=\mathrm{d} \omega_{k} / \mathrm{d} k$. It is related to the phase velocity $v_{k}$ of a plane wave as:

$$
v_{g}=v_{k}-\lambda_{k} \mathrm{~d} v_{k} / \mathrm{d} \lambda_{k} .
$$

Closely related is the center of the option wave-packet (the point of maximum amplitude), given by:

$s=t \mathrm{~d} \omega_{k} / \mathrm{d} k$.

The following quantum-motivated assertions can be stated:

1) Volatility $\sigma$ has dimension of financial action, or energy $\times$ time.

2) The total energy $E$ of an option wave-packet is (in the case of similar plane waves) given by Planck's superposition of the energies $E_{k}$ of $n$ individual waves:

$$
E=n \sigma \omega_{k}=\frac{n}{2}(\sigma k)^{2},
$$

where $L=n \sigma$ denotes the angular momentum of the option wave-packet, representing the shift between its growth and decay, and vice versa.

3) The average energy $\langle E\rangle$ of an option wave-packet is given by Boltzmann's partition function:

$$
\langle E\rangle=\frac{\sum_{n=0}^{\infty} n E_{k} \mathrm{e}^{-\frac{n E_{k}}{b T}}}{\sum_{n=0}^{\infty} \mathrm{e}^{-\frac{n E_{k}}{b T}}}=\frac{E_{k}}{\mathrm{e}^{\frac{E_{k}}{b T}}-1},
$$

where $b$ is the Boltzmann-like kinetic constant and $T$ is the market temperature.

4) The energy form of the Schrödinger Equation (18) reads: $E \psi=\mathrm{i} \sigma \partial_{t} \psi$.

5) The eigenvalue equation for the Hamiltonian operator $\hat{H}$ is the stationary Schrödinger equation:

$$
\hat{H} \varphi(s)=E \varphi(s), \quad \text { or } \quad E \varphi(s)=-\frac{\sigma^{2}}{2} \partial_{s s} \varphi(s),
$$

which is just another form of the harmonic oscillator (20). It has oscillatory solutions of the form:

$$
\varphi_{E}(s)=c_{1} \mathrm{e}^{\frac{\mathrm{i}}{\sigma} \sqrt{2 E_{k}} s}+c_{2} \mathrm{e}^{-\frac{\mathrm{i}}{\sigma} \sqrt{2 E_{k}} s},
$$

called energy eigen-states with energies $E_{k}$ and denoted by:

$$
\hat{H} \varphi_{E}(s)=E_{k} \varphi_{E}(s) .
$$

The Black-Scholes put and call options have been fitted with the quantum PDFs (see Figures 5 and 6) given by the absolute square of (21) with $n=7$ and $n=3$, respectively. Using supervised Levenberg-Marquardt algorithm and Mathematica 7, the following coefficients were obtained for the Black-Scholes put option: 


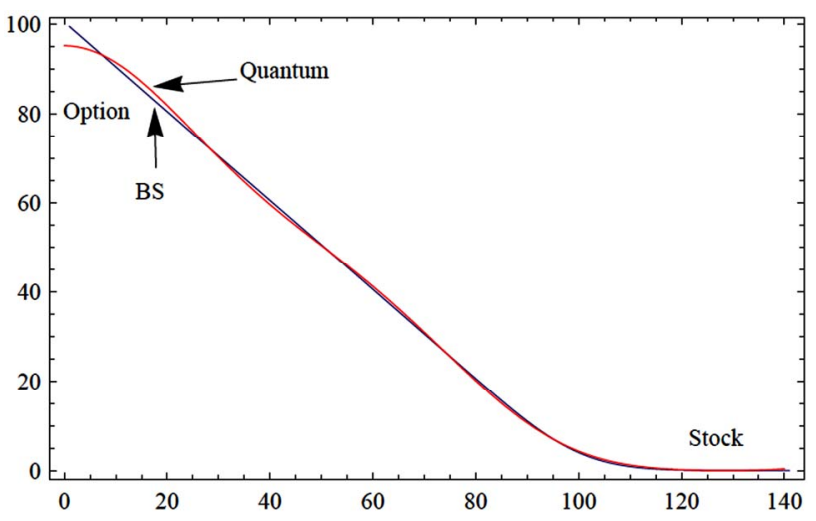

Figure 5. Fitting the Black-Scholes put option with the quantum PDF given by the absolute square of (21) with $n=7$.

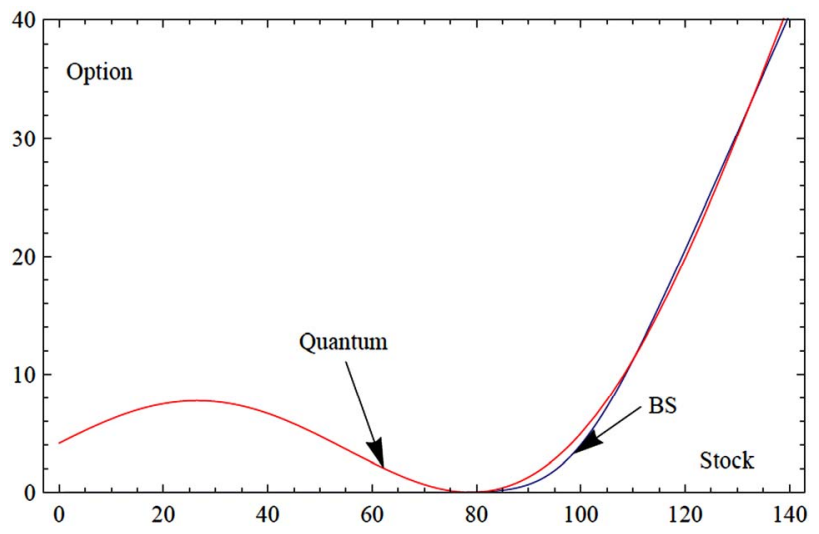

Figure 6. Fitting the Black-Scholes call option with the quantum PDF given by the absolute square of (21) with $n=3$. Note that fit is good in the realistic stock region: $s \in[75,140]$.

$\sigma^{*}=-0.0031891, t^{*}=-0.0031891$,

$k_{1}=2.62771, k_{2}=2.62777, k_{3}=2.65402$,

$k_{4}=2.61118, k_{5}=2.64104, k_{6}=2.54737, k_{7}=2.62778$,

$c_{1}=1.26632, c_{2}=1.26517$,

$c_{3}=2.74379, c_{4}=1.35495, c_{5}=1.59586$,

$c_{6}=0.263832, c_{7}=1.26779$,

with $\sigma_{B S}=-94.0705 \sigma^{*}, t_{B S}=-31.3568 t^{*}$.

Using the same algorithm, the following coefficients were obtained for the Black-Scholes call option:

${ }^{6}$ An example of a more general Gaussian wave-packet solution of (18) is given by:

$$
\psi(s, t)=\sqrt{\frac{\sqrt{a / \pi}}{1+\mathrm{i} a t}} \exp \left(\frac{-\frac{1}{2} a\left(s-s_{0}\right)^{2}-\frac{\mathrm{i}}{2} p_{0}^{2} t+\mathrm{i} p_{0}\left(s-s_{0}\right)}{1+\mathrm{i} a t}\right),
$$

where $s_{0}, p_{0}$ are initial stock-price and average momentum, while $a$ is the width of the Gaussian. At time $t=0$ the 'particle' is at rest around $s=0$, its average momentum $p_{0}=0$. The wave function spreads with time while its maximum decreases and stays put at the origin. At time $-t$ the wave packet is the complex-conjugate of the wave-packet at time $t$.

$$
\begin{aligned}
& \sigma^{*}=-11.9245, t^{*}=-11.9245, \\
& k_{1}=0.851858, k_{2}=0.832409, \\
& k_{3}=0.872061, c_{1}=2.9004, \\
& c_{2}=2.72592, c_{3}=2.93291, \\
& \text { with } \sigma_{B S}-0.0251583 \sigma^{*}, t=-0.00838609 t^{*} .
\end{aligned}
$$

Now, given some initial option wave function,

$$
\psi(s, 0)=\psi_{0}(s)
$$

a solution to the initial-value problem for the linear Schrödinger Equation (18) is, in terms of the pair of Fourier transforms $\left(\mathcal{F}, \mathcal{F}^{-1}\right)$, given by (see [42])

$$
\begin{aligned}
& \psi(s, t) \\
= & \mathcal{F}^{-1}\left[\mathrm{e}^{-\mathrm{i} \omega t} \mathcal{F}\left(\psi_{0}\right)\right]=\mathcal{F}^{-1}\left[\mathrm{e}^{-\mathrm{i} \frac{\sigma k^{2}}{2} t} \mathcal{F}\left(\psi_{0}\right)\right] .
\end{aligned}
$$

For example (see [42]), suppose we have an initial option wave-function at time $t=0$ given by the complexvalued Gaussian function:

$$
\psi(s, 0)=\mathrm{e}^{-a s^{2} / 2} \mathrm{e}^{\mathrm{i} \sigma k s},
$$

where $a$ is the width of the Gaussian, while $p$ is the average momentum of the wave. Its Fourier transform, $\hat{\psi}_{0}(k)=\mathcal{F}[\psi(s, 0)]$, is given by

$$
\hat{\psi}_{0}(k)=\frac{\mathrm{e}^{-\frac{(k-p)^{2}}{2 a}}}{\sqrt{a}} .
$$

The solution at time $t$ of the initial value problem is given by

$$
\psi(s, t)=\frac{1}{\sqrt{2 \pi a}} \int_{-\infty}^{+\infty} \mathrm{e}^{\mathrm{i}\left(k s-\frac{\sigma k^{2}}{2} t\right)} \mathrm{e}^{-\frac{a(k-p)^{2}}{2 a}} \mathrm{~d} k,
$$

which, after some algebra becomes

$$
\psi(s, t)=\frac{\exp \left(-\frac{a s^{2}-2 \mathrm{isp}+\mathrm{i} p^{2} t}{2(1+\mathrm{i} a t)}\right)}{\sqrt{1+\mathrm{i} a t}}, \quad(\text { with } p=\sigma k) .
$$

As a simpler example, ${ }^{6}$ if we have an initial option wavefunction given by the real-valued Gaussian function,

$$
\psi(s, 0)=\frac{\mathrm{e}^{-s^{2 / 2}}}{\sqrt[4]{\pi}},
$$

the solution of (18) is given by the complex-valued $\psi$ function,

$$
\psi(s, t)=\frac{\exp \left(-\frac{s^{2}}{2(1+\mathrm{i} t)}\right)}{\sqrt[4]{\pi} \sqrt{1+\mathrm{it}}} .
$$


From (22) it follows that a stationary option wave-packet is given by:

$$
\varphi(s)=\frac{1}{\sqrt{2 \pi}} \int_{-\infty}^{+\infty} \mathrm{e}^{\frac{\mathrm{i}}{\sigma} k s} \hat{\psi}(k) \mathrm{d} k, \text { where } \hat{\psi}(k)=\mathcal{F}[\varphi(s)] \text {. }
$$

As $|\varphi(s)|^{2}$ is the stationary stock PDF, we can calculate the expectation values of the stock and the wave number of the whole option wave-packet, consisting of $n$ measured plane waves, as:

$$
\langle s\rangle=\int_{-\infty}^{+\infty} s|\varphi(s)|^{2} \mathrm{~d} s \text { and }\langle k\rangle=\int_{-\infty}^{+\infty} k|\hat{\psi}(k)|^{2} \mathrm{~d} k .
$$

The recordings of $n$ individual option plane waves (19) will be scattered around the mean values (23). The width of the distribution of the recorded $s$ - and $k$-values are uncertainties $\Delta s$ and $\Delta k$, respectively. They satisfy the Heisenberg-type uncertainty relation:

$$
\Delta s \Delta k \geq \frac{n}{2}
$$

which imply the similar relation for the total option energy and time:

$$
\Delta E \Delta t \geq \frac{n}{2}
$$

\section{A New Stock-Market Research Program}

Based on the above wave stock-market analysis, I propose a new financial research program as follows.

Firstly, define the general adaptive wave model for option pricing evolution as a (linear) combination of the previously defined particular solutions to the adaptive NLS-Equation (5). The three wave-components of this general model are:

1) the linear wave packet $\Delta \psi_{\text {packet }}(s, t)$, given by (21);

2 ) the shock-wave $\psi_{\text {shock }}(s, t)$, given by (10); and

$3)$ the soliton $\psi_{\text {soliton }}(s, t)$, given by (12).

Formally, the general adaptive wave model is defined by:

$$
\begin{aligned}
\psi_{\text {general }}(s, t) & =A_{1} \sum_{i=0}^{n} c_{i} \mathrm{e}^{\mathrm{i}\left(k s-\omega_{k} t\right)} \\
& \pm A_{2} \sqrt{\frac{-\sigma}{\beta}} \tanh (s-\sigma k t) \mathrm{e}^{\mathrm{i}\left[k s-\frac{1}{2} \sigma t\left(2+k^{2}\right)\right]} \\
& \pm A_{3} \sqrt{\frac{\sigma}{\beta}} \operatorname{sech}(s-\sigma k t) \mathrm{e}^{\mathrm{i}\left[k s-\frac{1}{2} \sigma t\left(k^{2}-1\right)\right]}
\end{aligned}
$$

where $A_{i},(i=1, \cdots, 5)$ denote adaptive amplitudes of the three waves, while the other parameters are defined in the previous section.

Secondly, we need to find the most representative financial index or contemporary markets data that clearly show in their evolution both the efficient markets hypothesis [13] and adaptive markets hypothesis [14]. Once we find such a representative data, we need to fit it using our general wave Model (24) and the powerful Levenberg-Marquardt fitting algorithm. I remark here that, based on my empirical experience, the general wave Model (24) is capable of fitting any financial data, provided we use appropriate number of fitting coefficients (see [10] for technical details).

Once we have successfully fitted the most representative market data we will have a model that can be used for prediction of many possible outcomes of the current global financial storm.

\section{Conclusions}

I have proposed an adaptive-wave alternative to the standard Black-Scholes option pricing model. The new model, philosophically founded on adaptive markets hypothesis $[13,14]$ and Elliott wave market theory $[15,16]$, describes adaptively controlled Brownian market behavior. Two approaches have been proposed: 1) a nonlinear one based on the adaptive NLS (solved by means of Jacobi elliptic functions) and the adaptive Manakov system (of two coupled NLS equations); 2) a linear quantum-mechanical one based on the free-particle Schrödinger equation and de Broglie's plane waves. For the purpose of fitting the Black-Scholes data, the Levenberg-Marquardt algorithm was used.

The presented adaptive and quantum wave models are spatio-temporal dynamical systems of much higher complexity [25] then the Black-Scholes model. This makes the new wave models harder to analyze, but at the same time, their immense variety is potentially much closer to the real financial market complexity, especially at the time of financial crisis.

\section{References}

[1] F. Black and M. Scholes, "The Pricing of Options and Corporate Liabilities," Journal of Political Economy, Vol. 81, No. 3, 1973, pp. 637-659. doi:10.1086/260062

[2] R. C. Merton, "Theory of Rational Option Pricing," The Bell Journal of Economics and Management Science, Vol. 4, No. 1, 1973, pp. 141-183. doi:10.2307/3003143

[3] M. F. M. Osborne, "Brownian Motion in the Stock Market," Operations Research, Vol. 7, No. 2, 1959, pp. 145 173. doi:10.1287/opre.7.2.145

[4] K. Itô, "On Stochastic Differential Equations," Memoirs of the American Mathematical Society, Vol. 4, 1951, pp. $1-51$.

[5] J. Perello, J. M. Porra, M. Montero and J. Masoliver, Black-Scholes Option Pricing within Itô and Stratonovich Conventions, Physica A: Statistical Mechanics and Its 
Applications, Vol. 278, No. 1-2, 2000, pp. 260-274. doi:10.1016/S0378-4371(99)00612-3

[6] C. W. Gardiner, "Handbook of Stochastic Methods," Springer, Berlin, 1983.

[7] J. Voit, "The Statistical Mechanics of Financial Markets," Springer, Berlin, 2005.

[8] R. L. Stratonovich, "A New Representation for Stochastic Integrals and Equations," SIAM Journal on Control and Optimization, Vol. 4, No. 2, 1966, pp. 362-371. doi:10.1137/0304028

[9] M. Kelly, "Black-Scholes Option Model \& European Option Greeks," The Wolfram Demonstrations Project, 2009. http://demonstrations.wolfram.com/EuropeanOptionGree ks

[10] V. Ivancevic, "Adaptive-Wave Alternative for the BlackScholes Option Pricing Model," Cognitive Computation, Vol. 2, No. 1, 2010, pp. 17-30.

[11] V. Ivancevic and T. Ivancevic, "Complex Dynamics: Advanced System Dynamics in Complex Variables," Springer, Dordrecht, 2007.

[12] V. Ivancevic and T. Ivancevic, "Quantum Leap: From Dirac and Feynman, Across the Universe, to Human Body and Mind," World Scientific, Singapore, 2008. doi:10.1142/9789812819284

[13] A. W. Lo and J. Portf, "The Adaptive Markets Hypothesis: Market Efficiency from an Evolutionary Perspective," Journal of Portfolio Management, Vol. 30, 2004, pp. $15-29$

[14] A. W. Lo and J. Inves, "Reconciling Efficient Markets with Behavioral Finance: The Adaptive Markets Hypothesis," Journal of Investment Consulting, Vol. 7, 2005, pp. 21-44.

[15] A. J. Frost and R. R. Prechter Jr., "Elliott Wave Principle: Key to Market Behavior," 10th Edition, Wiley, New York, 1978

[16] P. Steven, “Applying Elliott Wave Theory Profitably,” Wiley, New York, 2003.

[17] V. Ivancevic and E. Aidman, "Life-Space Foam: A Medium for Motivational and Cognitive Dynamics," Physica A: Statistical Mechanics and its Applications, Vol. 382, No. 2, 2007, pp. 616-630. doi:10.1016/j.physa.2007.04.025

[18] V. Ivancevic and T. Ivancevic, "Quantum Neural Computation," Springer, Berlin, 2009.

[19] H. Kleinert and H. Kleinert, "Path Integrals in Quantum Mechanics, Statistics, Polymer Physics, and Financial Markets," 3rd Edition, World Scientific, Singapore, 2002.

[20] S. Liu, Z. Fu, S. Liu and Q. Zhao, "Jacobi Elliptic Function Expansion Method and Periodic Wave Solutions of Nonlinear Wave Equations," Physics Letters A, Vol. 289, No. 1-2, 2001, pp. 69-74. doi:10.1016/S0375-9601(01)00580-1

[21] G.-T. Liu and T.-Y. Fan, "New Applications of Developed Jacobi Elliptic Function Expansion Methods," Physics Letters A, Vol. 345, No. 1-3, 2005, pp. 161-166. doi:10.1016/j.physleta.2005.07.034
[22] M. Abramowitz and I. A. Stegun, Eds., "Jacobian Elliptic Functions and Theta Functions. Chapter 16 in Handbook of Mathematical Functions with Formulas, Graphs, and Mathematical Tables," 9th Edition, Dover, New York, 1972, pp. 567-581.

[23] V. Ivancevic and T. Ivancevic," Neuro-Fuzzy Associative Machinery for Comprehensive Brain and Cognition Modelling," Springer, Berlin, 2007. doi:10.1007/978-3-540-48396-0

[24] V. Ivancevic and T. Ivancevic, "Computational Mind: A Complex Dynamics Perspective," Springer, Berlin, 2007. doi:10.1007/978-3-540-71561-0

[25] V. Ivancevic and T. Ivancevic, "Complex Nonlinearity: Chaos, Phase Transitions, Topology Change and Path Integrals," Springer, Berlin, 2008.

[26] F. Black, "Studies of Stock Price Volatility Changes," Proceedings of the 1976 Meetings of the American Statistical Association, Business and Economics Statistics Section, 1976, pp. 177-181.

[27] H. E. Roman, M. Porto and C. Dose, "Skewness, LongTime Memory, and Nonstationarity: Application to Leverage Effect in Financial Time Series," Europhysics Letters, Vol. 84, No. 2, 2008, pp. 28001-28006.

[28] B. Kosko, "Bidirectional Associative Memories," IEEE Transactions on Systems, Man and Cybernetics, Vol. 18, No. 1, 1988, pp. 49-60. doi:10.1109/21.87054

[29] B. Kosko, "Neural Networks, Fuzzy Systems, A Dynamical Systems Approach to Machine Intelligence," Prentice Hall, Upper Saddle River, 1992.

[30] S.-H. Hanm, I. G. Koh, "Stability of Neural Networks and Solitons of Field Theory," Physical Review E, Vol. 60, No. 6, 1999, pp. 7608-7611. doi:10.1103/PhysRevE.60.7608

[31] S. V. Manakov, "On the Theory of Two-Dimensional Stationary Self-Focusing of Electromagnetic Waves," Soviet Physics JETP, Vol. 38, p. 248.

[32] M. Haelterman and A. P. Sheppard, "Bifurcation Phenomena and Multiple Soliton-Bound States in Isotropic Kerr Media," Physical Review E, Vol. 49, No. 4, 1994, pp. 3376-3381. doi:10.1103/PhysRevE.49.3376

[33] J. Yang, "Classification of the Solitary Waves in Coupled Nonlinear Schrödinger Equations," Physica D: Nonlinear Phenomena, Vol. 108, No. 1-2, 1997, pp. 92-112. doi:10.1016/S0167-2789(97)82007-6

[34] D. J. Benney and A. C. Newell, "The propagation of Nonlinear Wave Envelops," Journal of Mathematical Physics, Vol. 46, 1967, pp. 133-139.

[35] V. E. Zakharov, S. V. Manakov, S. P. Novikov and L. P. Pitaevskii, "Soliton Theory: Inverse Scattering Method. Nauka, Moscow, 1980.

[36] A. Hasegawa and Y. Kodama, "Solitons in Optical Communications," Clarendon, Oxford, 1995.

[37] R. Radhakrishnan, M. Lakshmanan and J. Hietarinta, "Inelastic Collision and Switching of Coupled Bright Solitons in Optical Fibers," Physical Review E, Vol. 56, No. 2, 1997, pp. 2213-2216. 
doi:10.1103/PhysRevE.56.2213

[38] G. Agrawal, "Nonlinear Fiber Optics," 3rd Edition, Academic Press, San Diego, 2001.

[39] J. Yang, "Interactions of Vector Solitons," Physical Review E, Vol. 64, No. 2, 2001, pp. 026607-026623. doi:10.1103/PhysRevE.64.026607

[40] J. Elgin, V. Enolski and A. Its, "Effective Integration of the Nonlinear Vector Schrödinger Equation," Physica D: Nonlinear Phenomena, Vol. 225, No. 2, 2007, pp. $127-$ 152. doi:10.1016/j.physd.2006.10.005

[41] D. J. Griffiths, "Introduction to Quantum Mechanics,' 2nd Edition, Pearson Educ. Int., (2005)

[42] B. Thaller, "Visual Quantum Mechanics," Springer, New York, 2000. 